\title{
Vers une nouvelle iconologie médiévale
}

À propos de...Gil Bartholeyns, P.-O. Dittmar \& Vincent Jolivet, Image et transgression au Moyen Âge, Paris, PUF, 2008. Jérôme Baschet, L'Iconographie médiévale, Paris, Gallimard, 2008. Olivier Boulnois, Audelà de l'image. Une archéologie du visuel au Moyen Âge. $\mathrm{V}^{\mathrm{e}}-\mathrm{XVI}^{\mathrm{e}}$ siècle, Paris, Seuil, 2008. Jean Wirth, L'Image à l'époque gothique (1140-1280), Paris, Cerf, 2008.

Towards a New Medieval iconology

\section{Thomas Golsenne}

\section{(2) OpenEdition}

\section{Journals}

Édition électronique

URL : https://journals.openedition.org/tc/4014

DOI : $10.4000 /$ tc. 4014

ISSN : 1952-420X

Éditeur

Éditions de l'EHESS

Édition imprimée

Date de publication : 31 décembre 2008

Pagination : 284-297

ISSN : 0248-6016

Référence électronique

Thomas Golsenne, "Vers une nouvelle iconologie médiévale», Techniques \& Culture [En ligne], 50 |

2008, mis en ligne le 30 novembre 2009, consulté le 29 septembre 2022. URL : http://

journals.openedition.org/tc/4014; DOI : https://doi.org/10.4000/tc.4014 


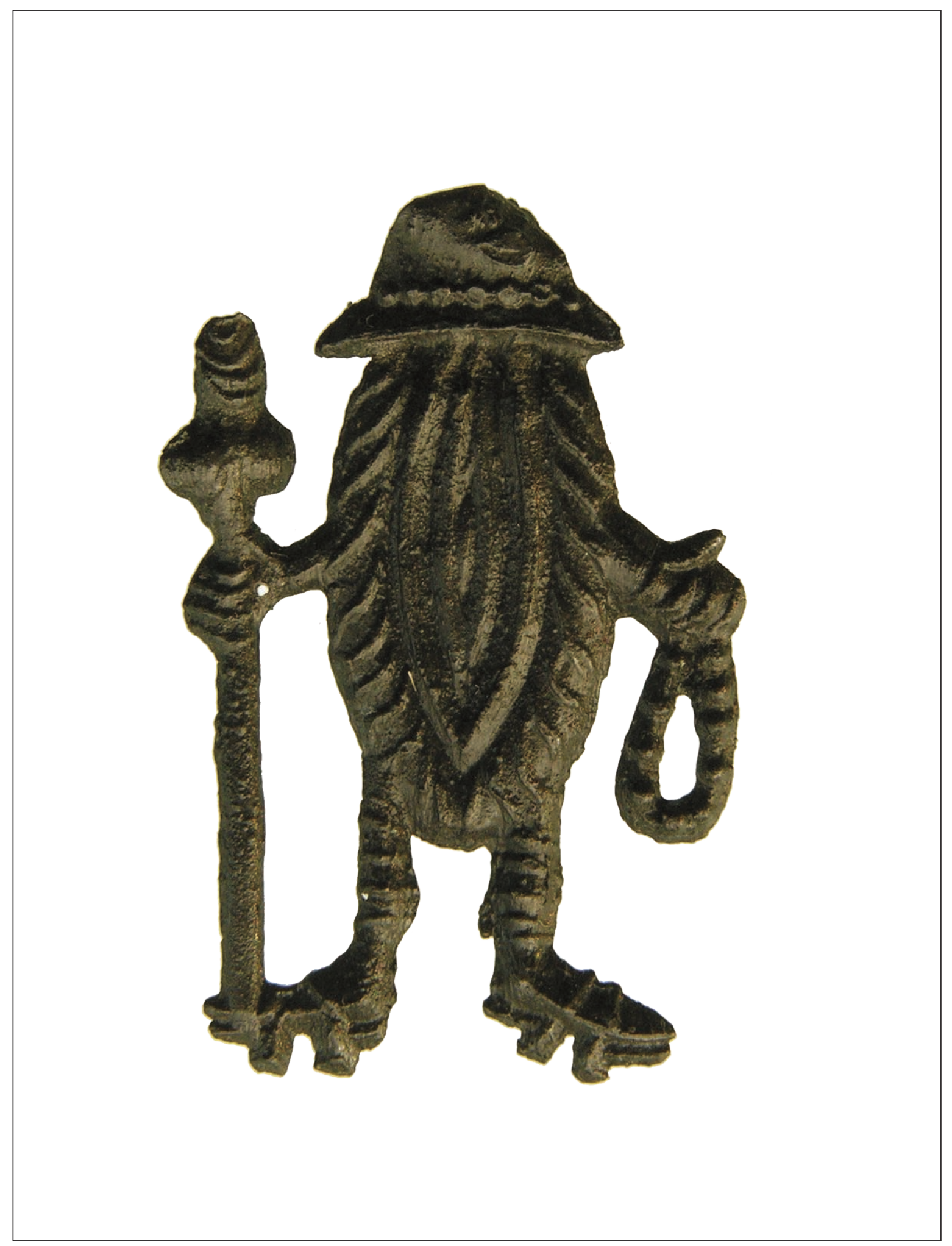




\title{
VERS UNE NOUVELLE ICONOLOGIE MÉDIÉVALE
}

\author{
À PROPOS DE... Gil Bartholeyns, Pierre-Olivier Dittmar \& Vincent Jolivet, Image \\ et transgression au Moyen Âge, Paris, PUF, 2008. Jérôme Baschet, Liconographie \\ médiévale, Paris, Gallimard, 2008. Olivier Boulnois, Au-delà de l'image. Une archéo- \\ logie du visuel au Moyen Âge. $V^{e}-X V I^{e}$ siècle, Paris, Éd. du Seuil, 2008. Jean Wirth, \\ L'Image à l'époque gothique (1140-1280), Paris, Éd. du Cerf, 2008.
}

Depuis une vingtaine d'années, le Moyen Âge joue un rôle de terrain d'expérimentation pour les nouvelles pratiques de recherches historiques et esthétiques. C'est que la reconfiguration récente de l'histoire de l'art s'est organisée autour d'une remise en question de son concept fondateur: l'art lui-même. Les historiens n'utilisaient plus depuis l'iconologie d'Erwin Panofsky les ouvres d'art comme des monuments formels qui auraient leur propre histoire, mais comme des documents visuels fournissant des renseignements sur une époque ${ }^{1}$. L'étape suivante a consisté à historiciser la notion d'art elle-même, à rendre obsolète l'idée que l'art était une sphère d'activité propre à l'homme, qui faisait partie de sa nature et montrait de quoi l'esprit humain était capable. On découvrait que les peintures de Fra Angelico, les sculptures des cathédrales gothiques, les frises du Parthénon ou des temples d'Angkor ne faisaient partie de l'histoire de l'art que pour notre regard occidental moderne. À l'époque où elles étaient produites, ces œuvres - disaient les iconologues - n'étaient pas plus considérées comme de l'art qu'une chaise ou une chaussure. Bref, elles relevaient de l'artisanat. Leur valeur d'usage était supérieure à leur valeur esthétique ${ }^{2}$.

\section{« Vulve pèlerine au bâton phallique et au rosaire »}

Enseigne, 33 × 19 mm, Reimerswall, 13751425. Collection H. J. E. Beuningen, Inv. 2184 (tirée de Bartholeyns, Dittmar \& Jolivet : 112, fig. 24)

(Fig. 1) 

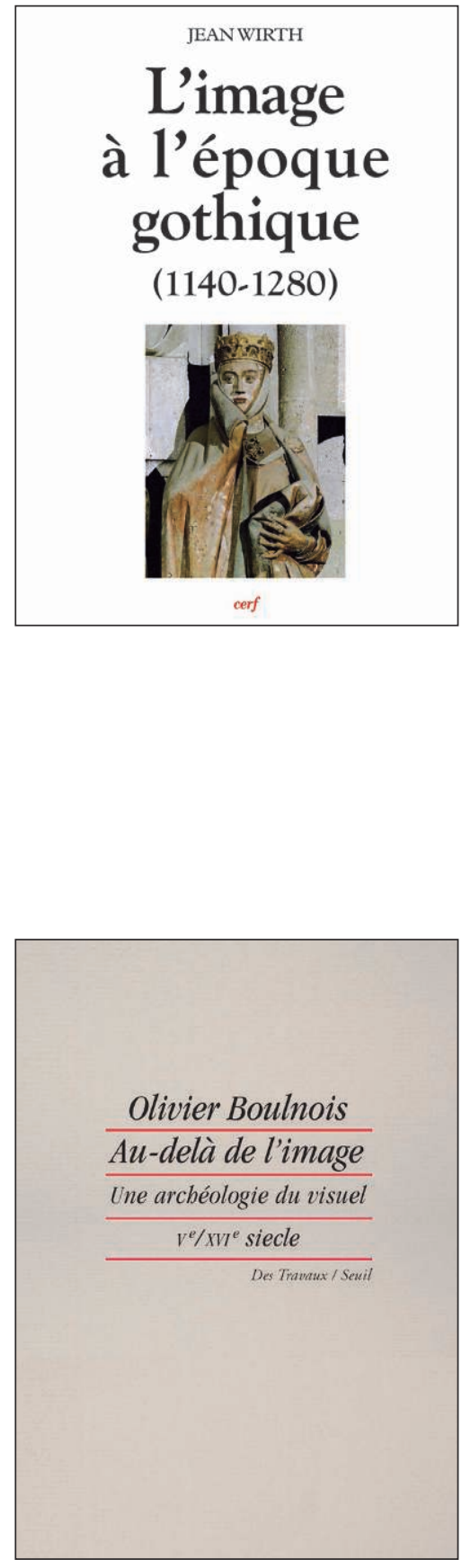

On en arrivait ainsi à penser que l'essence d'une peinture ou d'une sculpture (avant qu'elle ne soit considérée comme une ouvre d'art), c'était sa fonction (Didi-Huberman 1996), et que le travail du chercheur consistait à déterminer de quelles façons elle était utile.

On comprenait du même coup que l'art n'existait que du moment qu'un regard esthétique - un regard en quête de " plaisir désintéressé » - était mis en action; et l'on observait qu'avant la Renaissance, un tel regard n'était jamais porté sur les objets visuels. Ainsi, aussi bien Régis Debray, dans Vie et mort de l'image que Hans Belting dans Image et culte distinguaient avec force le regard religieux du regard esthétique et « l'ère de l'image » de «l'époque de l'art » (Debray 1992; Belting 1998). Notion au moins aussi (si ce n'est plus) complexe que celle d'art, l'image devenait ainsi le mot magique qui permettait d'en finir avec les anachronismes de l'œuvre d'art et de l'artiste et d'aborder les objets visuels de façon plus fidèle, plus historienne. Le Moyen Âge apparaissait du coup comme l'époque où la notion d'image était pensée dans toute sa richesse: pour décrire la culture médiévale, Jean-Claude Schmitt allait jusqu'à parler de « culture de l'imago » (Schmitt 1996). Dorénavant, il n'était plus possible de parler d'art médiéval, on allait étudier l'image médiévale ; on substituait à l'histoire de l'art une nouvelle « anthropologie des regards » (Didi-Huberman 1990 : 230). L'image réduisait l'œuvre d'art au statut de représentation, elle la ravalait au rang d'outil ; un outil abstrait, dont la signification était donnée par sa fonction supposée, et pas du tout par la technique employée pour le réaliser. Rares ont été les historiens de l'art à se servir d'André Leroi-Gourhan'3.

Mais peut-être qu'à force de dématérialiser l'objet visuel, de l'envisager du point de vue de sa réception en négligeant celui de sa production, on risquait de tomber dans l'excès inverse de celui de l'histoire de l'art à l'ancienne: en refusant toute dimension artistique aux images médiévales, on s'empêchait peut-être d'étudier comment, dans certaines circonstances, un regard esthétique intéressé avait pu, avant la Renaissance, tomber sur elles. Aussi, la tâche des historiens doit-elle se présenter aujourd'hui moins comme un dépassement que comme une refondation de l'histoire de l'art sur les bases d'une nouvelle iconologie, qui rééquilibre l'étude de l'image comme document et comme monument.

Quatre livres, parus récemment en France, poursuivent, chacun à leur manière, avec leurs objectifs propres, cette démarche ambitieuse de refondation de l'histoire de l'art à partir des images médiévales: L'Iconographie médiévale de Jérôme Baschet; Image et transgression au Moyen Âge de Gil Bartholeyns, Pierre-Olivier Dittmar \& Vincent Jolivet; Au-delà de l'image d'Olivier Boulnois; et L'Image à l'époque gothique de Jean Wirth. Ces livres répondent à des projets très différents.

Les auteurs d'Image et transgression partent du constat que certaines images médiévales choquent le spectateur d'aujourd'hui. Mais elles n'étaient pas conçues pour la transgression, au contraire: ce qui 
nous paraît incompréhensible aujourd'hui, c'est que l'image médiévale enseigne négativement la morale, par des contre-exemples, des exemples du mal. Dans d'autres cas, c'est magiquement que les images médiévales du mal repoussent le mal (les démons) (Fig. 1). Les images réellement transgressives sont donc rares, au Moyen Âge, et leur étude en est d'autant plus précieuse.

Jérôme Baschet offre une proposition méthodologique générale, à partir de quelques exemples remarquables, un peu à la manière du Détail de Daniel Arasse (Arasse 2006). Il s'agit de questionner la nature de l'image médiévale et, à partir de là, de construire une méthode sur mesure pour en déterminer la signification. Celle-ci doit être trouvée dans la dialectique du fond et de la forme qui organise la « pensée figurative » médiévale.

Pour Olivier Boulnois, l'image est avant tout un thème fondamental de l'histoire de la pensée médiévale en Occident. Contrairement au monde byzantin où la théologie des images est indissociable du culte des icônes, la théorie de l'image dans le monde latin a peu de rapports avec les images matérielles. Limage est pensée par les théologiens comme une représentation mentale, et toute la question qui se pose, à travers l'histoire de la théologie, est de savoir s'il est possible de produire une représentation mentale du divin, c'est-à-dire d'accéder à Dieu à partir de l'image.

Jean Wirth se livre à une exploration détaillée de la culture visuelle des $\mathrm{XII}^{e}$ et XIII ${ }^{e}$ siècles, qui prend place au sein d'une trilogie, dont les deux autres volets sont L'Image à l'époque romane (1999) et L'Image à la fin du Moyen Âge (à paraître). Aussi à l'aise avec la scolastique de Thomas d'Aquin que le portail de la cathédrale de Chartres, Wirth déduit des orientations théologiques de l'époque les grandes tendances qui s'expriment à travers les images et le style gothiques : tendance à la réconciliation entre le monde et Dieu, au naturalisme et à la clarification sémiotique. La méthode de Wirth est celle qui se rapproche sans doute le plus de l'iconologie panofskienne; son livre témoigne donc de la vivacité d'une telle démarche $e^{4}$.

Malgré ces disparités, y compris en termes d'utilisation et de qualité des reproductions, les quatre ouvrages comportent d'indéniables similitudes qui manifestent leur synchronie; qui permettent, en outre, de les faire dialoguer.

Leur premier point commun tient dans l'utilisation commune du mot magique: l'image. D'une façon ou d'une autre, les auteurs rejettent tous le vocabulaire de l'histoire de l'art traditionnelle, le terme « œuvre d'art » en particulier. Pour Baschet: « la notion d'Art, forgée par l'Esthétique des XVIII e et XIX ${ }^{e}$ siècles, est inadaptée à l'étude du Moyen Âge » (Baschet 2008 : 16). Pour Boulnois: « le Moyen Âge ne connaît pas de statut éminent accordé à l'art (celui-ci n'apparaît qu'à la Renaissance), et encore moins aux beaux-arts (il faudra attendre le XVIII ${ }^{e}$ siècle); il ignore la notion d'iconologie ${ }^{5}$ - qui fige et modifie la pratique en la codifiant; enfin, dans l'image, il ne cherche nullement le plaisir esthétique, mais la connaissance de la vérité » (Boulnois 2008 : 17). Les auteurs d'Image et transgression différencient nettement l'image médiévale et l'œuvre d'art
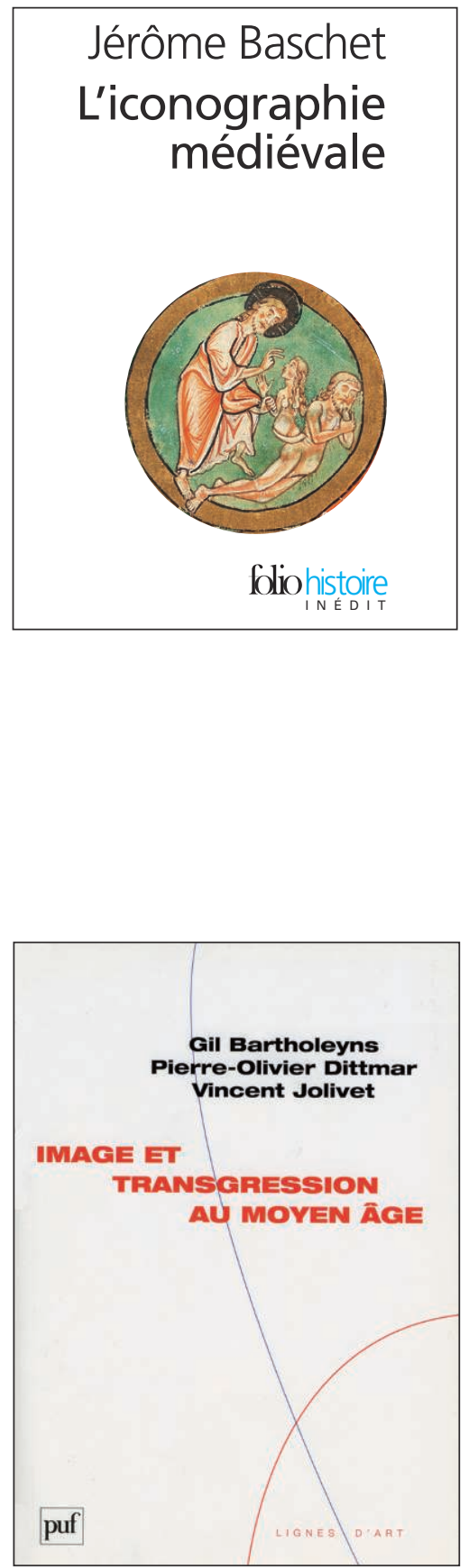


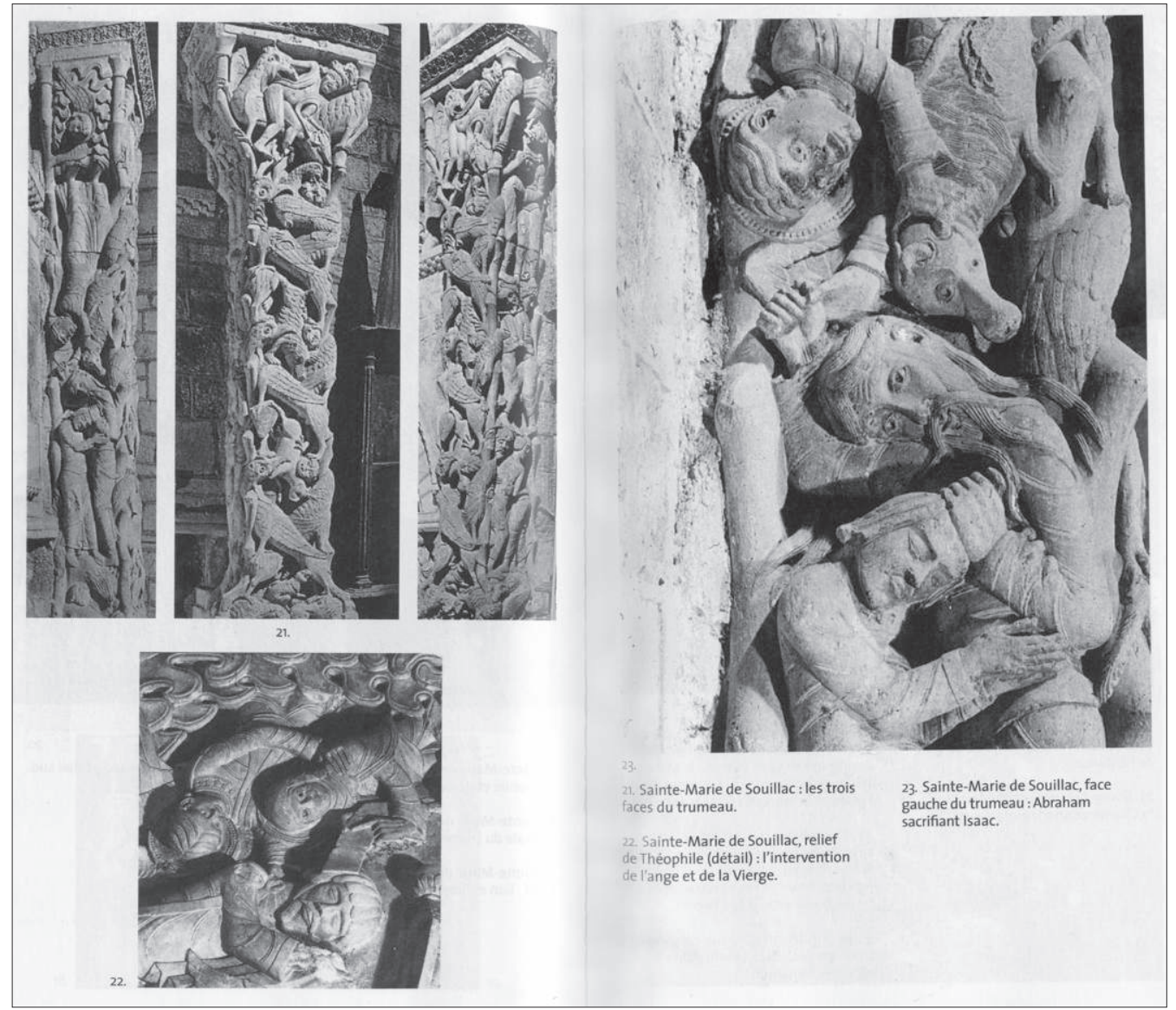

Sainte-Marie de Souillac, trumeau du porche central (tirée de Baschet 2008, figures 21-24).

(Fig. 1) moderne; cette distinction structure la thèse même du livre, puisque les auteurs peuvent opposer un régime a priori transgressif de l'œuvre d'art à l'époque moderne, à un régime a priori non transgressif de l'image médiévale. Wirth cependant se permet de parler de «l'art gothique» (Wirth 2008: 177) ou de « style » ou d'« artiste » mais reconnaît que « ce n'est pas sans scrupule que l'historien parle d'esthétique médiévale » (Ibid.: 117) et tout son chapitre "Art et création » est tendu vers l'idée qu'il n'y a pas, au XIII siècle, d' « autonomie du beau artistique » : la qualité d'une œuvre est avant tout morale ou didactique (Ibid. : 126).

Si le problème de l'image médiévale ne se situe pas au niveau esthétique, il se situe au niveau moral. Boulnois entend ainsi écrire, en parlant des images, un chapitre de "l'histoire de la vérité », projet très foucaldien (Boulnois 2008: 11). Pour Wirth, si les beaux-arts ou l'esthétique ne sont pas des domaines indépendants au Moyen Âge, c'est que les images y sont jugées comme des outils de connaissance. Les images permettent-elles de connaître les fins dernières, la Trinité, la vérité? Baschet, qui prend des exemples antérieurs, n'en adopte pas moins une approche de l'image assez similaire: la problématique morale est abordée dans son livre notamment à travers une sémantique de la hiérarchie. Les images sont adaptées à leur support, à leur fonction principale, à leur sujet; elles fournissent ainsi un 
ordre visuel qui peut trouver des équivalents dans la culture écrite, par exemple le trumeau de Souillac (Fig. 2), avec ses trois faces, qui représentent autant d'états de la relation entre l'homme et l'animal, l'homme entre le divin et le bestial... ${ }^{6}$ Les auteurs d'Image et transgression enfin, prennent le problème moral de l'image à bras-le-corps, puisque dans cet ouvrage la transgression y est avant tout pensée comme une transgression des mœurs: transgression sexuelle, transgression du bien et du mal.

Mais aborder l'image en termes de vérité ou de mensonge n'est pas très révolutionnaire, dans l'histoire européenne des idées: Boulnois a raison de commencer son livre avec Platon, car c'est bien le philosophe grec qui, et pour longtemps, a placé l'image sur le terrain moral de l'illusion. Ainsi, les théologiens médiévaux ont toujours plus ou moins situé la vérité des images en dehors d'elles-mêmes, les ont toujours à peu près envisagées sous l'angle de la représentation. Boulnois explique que, pour Thomas d'Aquin par exemple, la moralité d'une image ne se trouve pas dans les intentions de son auteur, mais dans ce qu'elle représente: l'image d'un diable est immorale et donc détestable, l'image du Christ est morale et donc vénérable (Boulnois 2008 : 269). D’où la tendance, chez les iconologues classiques, à considérer eux-mêmes les images comme des représentations, à s'intéresser surtout à leur sujet, plutôt qu'à leur style. Baschet le reconnaît: pour une partie - une partie seulement, j'y reviendrai -, l'image est « une représentation de l'univers et du monde social (et de la place qu'y occupent ceux qui prétendent à l'exercice d'une autorité) » (Baschet 2008: 36). Wirth n'hésite pas à dire, en conclusion de son chapitre sur « les moyens d'expression » gothiques, que « si l'évolution stylistique porte la marque des compromis et des ruptures, c'est dans l'iconographie qu'on lira le plus précisément l'équilibre instable des deux règnes » (Wirth 2008: 201): quand l'image devient du texte, elle devient plus riche d'enseignement historique.

Heureusement, les auteurs ne se contentent pas d'une ontologie de la représentation pour étudier les images médiévales, et la partie la plus novatrice de leurs recherches consiste précisément à remettre en cause, plus ou moins explicitement, ce modèle. Dans une page importante d'Image et transgression, les auteurs posent une question qui porte l'analyse des images dans une tout autre direction que celle de leur iconographie: «pourquoi les images [les représentations] de transgression sont demeurées des images morales du mal et par quels procédés elles ne sont pas transgressives » (Bartholeyns, Dittmar \& Jolivet 2008: 47). On voit là un paradoxe auquel Thomas d'Aquin aurait du mal à répondre, lui qui précisément affirme qu'une image du mal est une image mauvaise. C'est que les auteurs d'Image et transgression ne travaillent pas qu'à partir des textes normatifs, mais développent une approche pragmatique des images: comment ontelles été utilisées et reçues? Bref, quelle est leur efficacité? Ont-elles une puissance propre? Ceci suppose d'aborder l'image non plus seulement comme une représentation, mais comme une apparition : l'image produit un effet à partir du moment où elle existe, où elle est visible. Boulnois et 
Wirth savent également qu'au Moyen Âge, l'image n'était pas pensée et utilisée seulement comme une représentation. Dans plusieurs passages de son ouvrage, le premier parle de la « vénération des images », notamment dans le monde byzantin, où la croyance en la présence des saints, de la Vierge et du Christ dans les icônes est relayée par une théologie de l'Incarnation. Wirth remarque qu'au cours de sa période, le « culte » des images prospère et qu'il est même justifié par des théologiens de haut vol, comme Thomas d'Aquin. Ainsi croit-on l'époque douée d'une certaine «puissance » interne, virtus ou numen.

Mais Baschet, Bartholeyns, Dittmar et Jolivet vont plus loin, en distinguant la «puissance » mythique des images (celle à laquelle croient les fidèles du Moyen Âge face à la statue de la Vierge, au Crucifix) et leur « efficacité » historique, celle qui se vérifie dans les témoignages, les documents de l'époque, et qui s'exerce à travers un dispositif. C'est Baschet qui explicite le mieux cette position, dans le premier chapitre de son livre: « il est temps d'observer que l'image n'a, par elle-même, nul pouvoir. Son efficacité tient bien plutôt au jeu des relations idéelles et matérielles qui la traversent et qu'elle configure. » (Baschet 2008 : 50). Ainsi l'efficacité des images se manifeste-t-elle de façon très diverse: usages apotropaïques, votifs, dons d'images et dons à l'image, accompagnement d'un faire social, etc. Baschet va jusqu'à affirmer que ce qui rend l'image vraiment efficace, c'est sa présence; en d'autres termes, elle n'a même pas besoin d'être visible pour agir, comme dans le cas des images qui accompagnent le défunt dans la tombe (Ibid. : 55-56). Aussi, dit Baschet, l'image médiévale n'est-elle jamais pure représentation: elle est aussi, toujours, un objet. Soit elle est attachée à un support (les fresques, les statues des portails, les chapiteaux, les enluminures), soit elle est elle-même un objet (les statues-reliquaires). Son objectalité et son caractère représentationnel sont plus ou moins mis en valeur; en tout cas, ils produisent tous deux des effets et du sens (Ibid. : 62-63).

Critiquant tous plus ou moins le modèle de l'image-représentation, les quatre livres partagent la volonté de dépasser l'ancienne iconologie - ou tout du moins sa version française, l'iconographie d'Émile Mâle, qui, à la fin du XIX siècle, recueillit suffisamment d'informations sur les images médiévales pour que ses livres soient toujours considérés comme une base indispensable (Mâle : 1924, 1948 cités par Baschet et Wirth). En fait, l'iconographie de Mâle ressemble beaucoup à l'iconologie de Panofsky: il s'agit avant tout d'identifier les thèmes, les histoires, les symboles que les images médiévales illustrent, parfois de façon obscure. Il s'agit ensuite d'en déduire une leçon sur la «mentalité générale » de l'époque: culte marial, chasteté du regard médiéval, etc. Limage apparaît, chez Mâle, comme un document à traduire, et sa fonction supposée est de servir d'écriture aux illettrés. Contre cette conception inféodée de l'image à l'écrit, Baschet est celui qui s'élève le plus fortement. Elle est liée, selon lui, à une trop grande confiance de Mâle dans le discours des clercs et à son désintérêt 
pour toutes les autres formes d'utilisation des images qui précisément, contrevenaient au modèle institué par le pape Grégoire le Grand : celui d'une fonction didactique des images, bien détaillée par Boulnois (82-95). Elle est le résultat, enfin, d'une approche simpliste des images, réduites à des messages codés, en ignorant la polysémie propre au langage visuel (Baschet 2008: 150-160).

La nouvelle iconologie, que ces livres présentent d'un point de vue différent, garde de l'ancienne l'idée que les images sont toujours plus ou moins rapportées à des textes, au Moyen Âge; mais ce rapport est maintenant pensé de façon plus complexe, plus ouvert. Aussi la réforme entreprise par les auteurs vise-t-elle avant tout la sémiologie des images, la façon dont elles véhiculent ou produisent du sens. Les quatre ouvrages donnent chacun leur réponse à la question: en quoi consiste le sens d'une image médiévale? Mais, au lieu de s'en tenir à une réponse iconologique traditionnelle - dans ce qu'elle représente, ou bien dans sa fonction - la réponse est biaisée: les images sont étudiées non pas seulement pour leur contenu, comme le faisaient Mâle ou Panofsky, mais surtout pour la façon dont elles produisent du sens. C'est moins dans leur signification iconographique que dans leur fonctionnement que réside le sens des images médiévales. Par exemple, Boulnois insiste beaucoup, dans les textes théologiques, sur la dynamique visuelle de l'âme: il est acquis en effet depuis saint Augustin que l'âme s'élève vers Dieu à travers une succession de strates mentales, qui correspondent à différents types de visions: vision sensible, vision imaginaire, vision intellectuelle. Tout l'intérêt du livre consiste en ce qu'il montre très bien que les théologiens médiévaux n'ont pas beaucoup, contrairement à ce qu'on pourrait croire, retenu la leçon platonicienne de l'image-mensonge, mais ont plutôt considéré l'image comme un véhicule de l'âme (Fig. 3).

Ainsi, si l'homme doit « se dénuder de l'image » (c'est-à-dire quitter son enveloppe mortelle et corporelle de créature «à l'image de Dieu ») il doit aussi « entrer dans l'image » (c'est-à-dire redevenir "l'image de Dieu ») (Boulnois 2008 : 290-91). L'entreprise méthodologique de Baschet consiste précisément à proposer quelques pistes pour l'analyse dynamique ou relationnelle des images: une attention au lieu de l'image et à sa disposition dans l'espace, à la fois pour prendre en compte la position réelle de l'observateur dans le fonctionnement des images (Baschet accorde une notable importance à la question de «l'impression immédiate » face à la sensation colorée ou ornementale produite par
Sainte-Marie de Souillac « La Trinité invisible », Rotschild Canticles, Yale University, Beinecke Rare Book and Manuscript Library, Ms. 404, f. 40 r (tirée de Boulnois 2008,

ill. 3).

(Fig. 3). 
IMAGE ET TRANSGRESSION AU MOYEN ÂGE
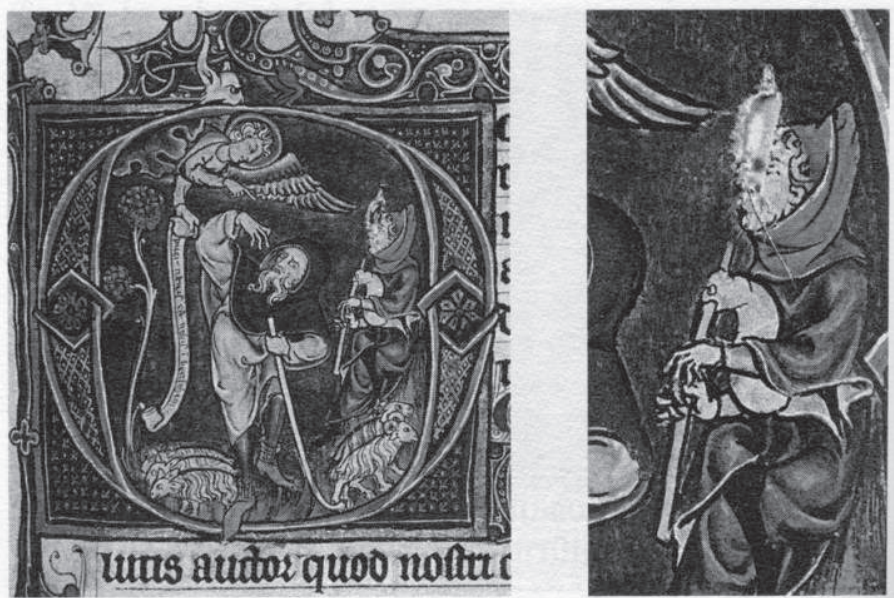

31. Annonce aux bergers. Livre d'heures à l'usage de Thérouanne, Artois, vers 1280 1290. Marseille, Bibliothèque municipale, ms. 111, f. 18.

de les poser sur la table de la salle à manger ${ }^{1}$.» Ainsi des images banales vont déranger en raison de leur association, de leur montage avec un contexte - un lieu, une autre image, un regard particulier - inadéquat selon les critères du moment. En fonction de la culture de chaque regardeur, des associations ou des proximités ont paru si inadéquates que des destructions s'en sont suivies. On peut alors chercher les rapports dans lesquels s'inscrivent les images détruites, voir à quoi elles sont associées.

Considérons trois possibilités de montages transgressifs.

- Il est arrivé qu'un imagier brouille lui-même la division fondamentale entre les différents espaces de la page médiévale, utilisant dans le «centre» une figure typique de la «marge ». C'est le cas dans l'exemple de l'annonce aux bergers que nous évoquions à l'instant (fig. 31). À côté du berger prin

1. M. DOUglas, De la souillure..., op. cit., p. 55.

exemple par un ensemble de fresques) et la structure interne des images: un cycle narratif ne signifie pas la même chose selon qu'il est disposé en bandes parallèles ou en boustrophédon; une distinction précieuse entre " ambiguité » et « ambivalence » (une image ambiguë souffre d'un défaut de sens, tandis qu'une image ambivalente fait preuve d'un surplus de sens); une mise en série des images, dans la durée et dans l'espace, afin de reconnaître les « hyperthèmes », les écarts et les exceptions à la norme. Wirth, qui lui aussi remarque la diversité et l'inventivité des images médiévales, a des passages très intéressants sur la question du « programme iconographique »: les iconologues, en général rationalistes et kantiens, sont toujours à l'affût de la plus grande cohérence possible quand ils regardent des images complexes, par exemple des portails de cathédrale; ils supposent toujours que le portail répond à une commande précise, que chaque figure a son rôle et sa fonction dans l'ensemble, bref que tout a été programmé par un clerc. Or ce qui marche peut-être pour les plafonds à fresque du XVII ${ }^{e}$ siècle est beaucoup plus difficile à prouver pour les ensembles iconographiques du $\mathrm{XIII}^{e}$; et, face aux bizarreries évidentes des portails de la cathédrale de Reims

BMVR, Marseille, Ms 111 fol 18. « Annonce aux bergers », Livre d'heures à l'usage de Thérouanne, Artois, v. 1280-90 (tirée de Bartholeyns, Dittmar \& Jolivet $2008: 140$ ).

(Fig. 4).
(Fig. 4), Wirth n'y voit pas un défaut de cohérence, ni l'application ratée d'un programme raté, mais plutôt un compromis entre un «bricolage » de sculptures provenant d'ateliers divers et produits sur de nombreuses années et un goût esthétique pour la varietas: « En d'autres termes, les commanditaires étaient peut-être moins épris de systèmes et plus esthètes que nous n'avons tendance à l'imaginer » (Wirth 2008 : 112-15).

Le modèle de composition par bricolage, familier aux anthropologues depuis La Pensée sauvage, est peut-être plus généralement admis que Wirth ne le dit. Boulnois montre très bien que l'idée de création humaine pure est jusqu'au XIV`e siècle (sauf chez certains marginaux de la pensée scolastique comme Guillaume d'Ockham et Nicolas de Cues) à peu près inconnue, et même interdite: seul Dieu crée à partir de rien. L'homme, pour sa part, se 
contente d'imiter; sa création est toujours seconde, son invention consiste toujours à extraire de la nature et à combiner des éléments qui lui seront utiles (Boulnois 2008 : 351-62). C'est la définition même du bricolage chez Lévi-Strauss. Autrement dit, une image est toujours une composition d'éléments déjà créés, d'objets trouvés; par extension, une image ne fonctionne jamais seule, c'est un ensemble ouvert qui se combine avec les éléments de son environnement. La théorie médiévale du bricolage peut être ainsi comparée avec profit au modèle du « montage » utilisé par Bartholeyns, Dittmar \& Jolivet. Comme au cinéma, les images médiévales sont toujours "montées », associées les unes aux autres, ou bien reliées à des textes; le sens transgressif ou pas des images dépend en fait du montage dans lequel elles s'inscrivent: ou bien c'est un montage "normatif », et dans ce cas il confère à une image de diable, de rapport sexuel impur ou de monstre un sens négatif de contre-modèle; ou bien c'est un montage « transgressif », où l'image même la plus innocente, parce qu'elle est mal raccordée, devient porteuse d'une puissance destructrice à l'égard de l'ordre esthético-moral en vigueur (c'est le cas par exemple de cette initiale représentant L'annonce aux bergers où le visage d'un berger qui joue de la levrette, une sorte de cornemuse qui ressemble vaguement aux organes génitaux masculins, a été gratté, comme si sa présence, dans une des parties les plus honorifiques de la page, était choquante, cf. Bartholeyns, Dittmar \& Jolivet 2008: 140-41, Fig. 4).

Si les images sont définitivement « bonnes à penser » pour les historiens, les quatre livres présentés ici montrent que le Moyen Âge est bon pour penser les images. Et ceci, pour une raison profonde, que Boulnois résume efficacement: "Soi-même comme une image, telle pourrait être la définition de l'anthropologie médiévale » (Boulnois 2008 : 266). Dieu n'a-t-il pas créé l'homme «à son image et à sa ressemblance »? Mais penser en ces termes l'anthropologie médiévale, c'est la penser en termes religieux. Et effectivement, la plupart des textes et des images invoqués par les auteurs sont tirés de la culture chrétienne. Chacun exclut l'interprétation profane (c'est-à-dire moderne?) de l'image médiévale. Dans Image et transgression, celle-ci consisterait à prouver que les images présentées étaient transgressives; or tout le livre est basé sur l'idée que ces images ne l'étaient pas aux yeux de ceux qui les ont conçues et dans les endroits pour lesquels elles étaient faites, c'est-à-dire les clercs et les églises. Wirth dément dès le départ une émancipation quelconque des laïcs et d'une sensibilité profane ${ }^{7}$. Baschet s'en prend à Meyer Schapiro qui voyait dans certains chapiteaux ou marges de manuscrits l'invention libre d'artistes dégagés de toute obligation religieuse (Baschet 2008 : 192). Boulnois oppose nettement le discours médiéval, religieux, sur les images, et le discours qui manifeste à partir de la Renaissance un regard profane sur les mêmes objets.

Pourtant, si la sphère profane ne s'est pas autonomisée au Moyen Âge comme elle l'est aujourd'hui, on peut dire qu'il y a du profane qui traverse le sacré durant cette période. De la même façon, Baschet reconnaît que 
s'il n'existe pas alors un domaine indépendant des beaux-arts, il n'y en a pas moins de l'art dans les images (Baschet 2008 : 16). La valeur esthétique des productions artisanales qui ornaient la basilique de Saint-Denis et la qualité du travail des orfèvres étaient reconnues par un esprit fort comme l'abbé Suger, au XII ${ }^{e}$ siècle. Son ennemi, Bernard de Clairvaux, partisan d'une plus grande sobriété dans la décoration des sanctuaires, voyait pour sa part dans les « belles difformités et les beautés difformes » des chapiteaux des cloîtres de son époque l'indice non d'une signification religieuse, mais d'une licence coupable des sculpteurs (Boulnois 2008 : 105-14). C'est dire que, positivement ou négativement, quelque chose comme le travail propre du sculpteur ou du peintre est reconnu au beau milieu du Moyen Âge, indépendamment ou à côté de la fonction morale ou de l'utilisation religieuse de leurs images. Cela se comprend: comme le dit Boulnois, il n'y a pas d'orthodoxie iconographique médiévale dans le monde latin. Cela ne signifie pas que « tout est permis » aux plasticiens médiévaux, comme Bartholeyns, Dittmar \& Jolivet le montrent amplement, mais que le respect des normes permet suffisamment de licence pour que les images médiévales se développent, en Occident, avec une richesse foisonnante. Aussi, si l'on recueille les données chronologiques fournies par les quatre ouvrages, on s'aperçoit d'un phénomène assez net global: la frontière entre le sacré et le profane a tendance à s'estomper à partir du XII siècle; malgré des réactions (saint Bernard, les protestants), cette tendance ne cesse de s'accroître avec le temps. Boulnois observe, sur le long terme, un passage, dans la théologie, de l'invisibilité totale du Père à son absolue visibilité, au XVe siècle (Ibid. : 447-48). La période étudiée par Wirth est particulièrement révélatrice à cet égard: l'âge gothique est marqué selon lui par un optimisme certain, qui se manifeste entre autres par le naturalisme des formes plastiques, montrant une vision positive, enchantée du monde, par une façon de glorifier les figures sacrées (la Vierge notamment) en leur donnant l'apparence fastueuse de rois et de reines mondaines (Wirth 2008 : 133-76). Sans doute cette imbrication du spirituel et du matériel, du visible et de l'invisible, est-elle un mouvement de fond qui commence avant le XIII siècle, comme les exemples donnés par Baschet le prouvent. Et qui, probablement, ne s'arrête pas avec la Renaissance; au contraire, celle-ci pourrait être considérée comme l'aboutissement de cette tendance (comme le montre ne serait-ce que la violence de l'iconoclasme protestant et des guerres de religion).

Bref, le Moyen Âge voit apparaître progressivement ce que Michel Maffesoli appelle un «matérialisme mystique », une conjonction du profane et du sacré, par laquelle s'explique l'importance cruciale des images dans la culture du bas Moyen Âge (Maffesoli 1990: 68) ${ }^{8}$. Non seulement intermédiaires, moyens, ou outils : les images au Moyen Âge peuvent être considérées comme des fins en soi. Elles sont les fruits d'une société qui les cultivent parce qu'elle les trouve bonnes pour sa santé. En termes moins métaphoriques: la prolifération des images et 
leur qualité sont cause et résultat direct du développement de la société médiévale dans le sens de cette spiritualisation de la matière et de cette matérialisation de l'esprit qui s'accentuent au cours des siècles. Une image médiévale n'est pas a priori profane ou religieuse: elle est les deux à la fois. Si l'image se voit consacrer une place primordiale dans la société médiévale, c'est que les sentiments qu'elle procure sont ceux que recherchent les hommes et les femmes du Moyen Âge pour vivre ensemble. Limage médiévale est un ciment social.

On mesurera ainsi l'écart que cette expérience médiévale de l'image entretient avec son avatar moderne, qui s'est construit au XIX siècle. Au temps des musées et des esthètes, la profonde expérience face à une œuvre d'art est celle qui soustrait le spectateur ravi au monde, à l'image de l'artiste qui s'est mis lui-même en marge de la société. Comme Bergotte qui meurt face à la Vue de Delft de Vermeer ${ }^{9}$. C'est dans ce régime que l'artiste est apparu comme le transgresseur des normes sociales bourgeoises: l'utilité, l'économie, la mesure; et certains sociologues, comme Nathalie Heinich, citée par Bartholeyns, Dittmar \& Jolivet, continuent à penser l'art contemporain sous cet angle. Mais on peut se demander si aujourd'hui l'art ne joue pas un autre rôle, plus proche que celui de l'image dévotionnelle dans la société médiévale. Un touriste moyen ne cherche plus à travers l'art l'expérience profonde et narcissique qui l'atteindra au cœur de son être individuel. Le touriste accomplit des sortes de pèlerinages culturels au terme desquels il est mis en présence du chef-d'œuvre, avec ses semblables (Amirou 1995). Certains regrettent la perte de qualité du regard que cette évolution récente implique ${ }^{10}$. Mais le vrai but de tels voyages ne serait-il pas de partager une expérience sensible, de se retrouver autour d'un patrimoine commun ${ }^{11}$ ? Il y a de quoi encore travailler sur ces bases; et peut-être que la comparaison avec le Moyen Âge, son culte des images et son dieu incarné, permettrait de mieux saisir l'étrangeté de notre «société des images » où Dieu semble faire son - inquiétant? - retour. 


\section{NOTES}

1. Sur la distinction entre monument et document dans l'iconologie, Panofsky 1969 : 37-38. Sur une utilisation dérivée de Panofsky de l'image comme document, voir par ex. Haskell 1993.

2. Panofsky pense encore que la meilleure ouvre d'art est celle qui équilibre son utilité et sa forme (1969 : 38-41); bref, il la pense comme un objet design. Mais des historiens plus récents se soucient bien peu de la qualité formelle des images.

3. Il y a des exceptions. Pour l'image ancienne, notons Georges Didi-Huberman et ses travaux sur l'empreinte (Didi-Huberman 1997). Pour le domaine récent, les recherches sur la photographie et le cinéma ne peuvent pas, le plus souvent, faire l'économie des questions technologiques.

4. Voir un compte rendu détaillé de l'ouvrage de Jean Wirth par Jérôme Baschet en ligne: http://www.editionspapiers.org/node/26.

5. Boulnois se réfère ici à l'« iconologie » non au sens de Panofsky, mais de Cesare Ripa, auteur d'une Iconologia à la fin du XVI e siècle qui se présente comme un recueil de symboles, d'allégories et de personnifications, visant à établir un code visuel universel.
6. « Le trumeau, qui est peut-être comme la porte de l'œuvre, indique qu'entrer dans l'édifice cultuel c'est franchir un seuil de vérité » (Baschet 2008: 219)

7. À propos du Roman de la Rose, puis de l'interprétation formaliste de Meyer Schapiro d'un texte complexe, (Wirth 2008 : 64), puis de l'interprétation littérale de scènes apparemment profanes de l'Ancien Testament, qui sont tout de suite interprétées en termes religieux, néotestamentaires, (Ibid. :86) : le profane est " péjoratif ».

8. Spécialiste de l'Italie de la fin du Moyen Âge, Richard Trexler y notait pour sa part la manifestation d'une « perception sensuelle » de Dieu (Trexler 1972: 9).

9. Sur le culte individualiste de l'art au XIX ${ }^{e}$ siècle, voir Belting 2003 : 300-305 sur Proust et Vermeer).

10. Voir les observations sans appel de Recht (2006: 25-27), plus réfléchies d'Aboudrar (2000 : 138-39), plus nuancées d'Arasse (2006 : 265-66).

11. Debray établit un parallélisme entre le culte médiéval des images de dévotion et le culte actuel des œuvres d'art dans les musées (Debray 1992 : 411-21). Mais déjà Riegl ([1903] 1984) associait nationalisme et culte des monuments. 


\section{RÉFERENCES}

Aboudrar, Bruno-Nassim, 2000, Nous n'irons plus au musée. Paris, Aubier.

Amirou, Rachid, 1995, Imaginaire touristique et sociabilité du voyage. Paris, PUF.

Arasse, Daniel, 2006, Histoires de peintures. Paris, Gallimard.

Bartholeyns, Gil, Dittmar, Pierre-Olivier \& Jolivet, Vincent, 2008, Image et transgression au Moyen Âge. Paris, PUF.

Baschet, Jérôme, 2008, L'Iconographie médiévale. Paris, Gallimard.

Belting, Hans, 1998, Image et culte. Une histoire de l'image avant l'époque de l'art. Paris, Éd. du Cerf.

Belting, Hans, 2003, Le Chef-d'œuvre invisible. Nîmes, J. Chambon.

Boulnois, Olivier, 2008, Au-delà de l’image. Une archéologie du visuel au Moyen Âge. Vé-XVIe siècle. Paris, Éd. du Seuil.

Debray, Régis, 1992, Vie et mort de l'image. Une histoire du regard en Occident. Paris, Gallimard.

Didi-Huberman, Georges, 1990, Devant l'image. Question posée aux fins de l'histoire de l'art. Paris, Éd. de Minuit.

Didi-Huberman, Georges, 1996, «Imitation, représentation, fonction. Remarques sur un mythe épistémologique », in J. Baschet et J.-C. Schmitt, (eds), L'Image. Fonctions et usages des images dans l'Occident médiéval. Paris, Cahiers du Léopard d'or 5 : 59-86.

Didi-Huberman, Georges, 1997, L'Empreinte. Catalogue de l'exposition au Centre Pompidou, 19 février19 mai 1997, Paris, Centre G. Pompidou (le texte « La ressemblance par contact » qui introduit le catalogue a été republié aux Éd. de Minuit en 2008)

Haskell, Francis, 1993, L'Historien et les images. Paris, Gallimard.

Maffesoli, Michel, 1990, Au creux des apparences. Pour une éthique de l'esthétique. Paris, Plon.

Mâle, Émile, 1924, L’Art religieux du XII siècle en France. Paris, A. Colin.

Mâle, Émile, 1948, L'Art religieux du XIII e siècle en France. Étude sur l'iconographie du Moyen Âge et sur ses sources d'inspiration. 8e éd.. Paris, A. Colin.

Panofsky, Erwin, 1969, «L'histoire de l'art est une discipline humaniste », in L'Euvre d'art et ses significations. Essais sur les arts visuels (Meaning in the visual arts, New York, Doubleday, 1955, trad. Marthe et Bernard Teyssèdre), Paris, Gallimard: 27-52.

Recht, Roland, 2006, À quoi sert l'histoire de l'art? Paris, Textuel.

Riegl, Aloïs, [1903] 1984, Le Culte moderne des monuments. Son essence et sa genèse. Paris, Éd. du Seuil.

Schmitt, Jean-Claude, 1996, « La culture de l'imago », in Annales HSS 1 : 3-36

Trexler, Richard, 1972, "Florentine religious expérience: the sacred image », in Studies in the Renaissance XIX: $7-41$

Wirth, Jean, 2008, L’Image à l'époque gothique (1140-1280). Paris, Éd. du Cerf. 